\title{
Aerobic Glycolysis Pathway Activation by Epstein-Barr Virus Latent Membrane Protein 1 in Nasopharyngeal Carcinoma
}

\author{
Yaacoub S, Zgheib H, Mammari N, Chelala H and Halabi MA* \\ Holy Family University, Department of Medical Sciences, Batroun, Lebanon
}

*Corresponding author: Mohamad Adnan HALABI, Holy Family University, Department of Medical Laboratory Sciences, Batroun, Lebanon, Tel: 0096171721832; Email: adnan.halaby@gmail.com

\section{Review Article \\ Volume 2 Issue 11}

Received Date: October 22, 2018

Published Date: November 02, 2018

DOI: $10.23880 /$ vij- 16000200

\section{Abstract}

Glucose, is a major source of energy to the cell growth and energy production. During tumors formation and cancer cell growth glycolysis significantly increases as well as glucose uptake and therefore increase biosynthesis, in addition to many more metabolic changes. These transformations are known as "Warburg effect" in presence of adequate oxygen (aerobic glycolysis).

LMP-1 regulates and promotes glycolysis through many pathways, genes, gene regulators, tumor suppressors, and enzymes such as PI3-K/Akt, Ras, mTOR, HIF-1 $\alpha$, c-Myc, NF-кB, p53, GSK3beta, FBW7, FGFR1, the M2 splice isoform of pyruvate kinase PKM2, PDHK1, Hexokinase 2 HK2, VHL, and mutations of isocitrate dehydrogenase 1 (IDH1), succinate dehydrogenase (SDH), and fumarate hydratase (FH).

The aim of this study is to analyze the most recent articles on how LMP1 can alter the signaling pathways of glycolysis in the nasopharyngeal carcinoma.

Keywords: Epstein-Barr Virus; Glycolysis; LMP1

\section{Introduction}

Epstein Bar Virus 'EBV' is a ubiquitous human herpesvirus, which infects almost the entire human adult population worldwide and with life-long persistence in the host. It is known to cause infectious mononucleosis and to be associated with several human malignancies, such as nasopharyngeal carcinoma (NPC), Burkitt's lymphoma (BL), mixed cellularity subtype Hodgkin's lymphoma (HL) and Extranodal natural killer/T-cell lymphoma (NKTL). EBV can be typed into A and B types based on large differences observed in nuclear protein genes including Epstein-Barr nuclear antigen type 2
(EBNA2). Type A is more prevalent in the developed world whereas type $B$ is encountered in equatorial Africa and Guinea. Type A EBV was predominant in NKTL in Korea, Japan and Malaysia (6), and more generally in Asia [1-5]. EBV can undergoes two types of infection, latent and lytic in order to achieve a persistent infection in human. The Latent Membrane Protein 1(LMP-1) is an essential viral protein for the maintenance of latent infection.

LMP1is a membrane protein of 386 amino acids [6], comprises of an amino acid cytoplasmic $\mathrm{N}$-terminus (amino acids 1-23), six hydrophobic alpha-helical 
transmembrane regions (amino acids 24-186), and a large cytoplasmic C-terminus tail (amino acids 187-386) [7,8]. The $\mathrm{N}$ - and $\mathrm{C}$ - terminal domains as well as the alphahelical transmembrane regions.

This structure makes LMP1a docking sites for signaling adaptor proteins, tumor necrosis factor receptor-associated factor (TRAF), and tumor necrosis factor receptor-associated death domain (TRADD) respectively activating a number of signaling pathways $[8,9]$. These pathways lead to the transformation of Bcells into cancerous or immortals due to the activation of the nuclear factor kappa nuclear factor-kappa B (NF-? B) which has been proven to be involved in the regulation of viral and cellular genes involved in proliferation and/or activation as well as protecting the cell from apoptosis by activating A20 gene [10]. CTAR3, which lies between CTAR1 and CTAR2, has been shown to recruit Janus kinase 3 (JAK3) which results in signaling transducer and activator of transcription type 3 (STAT3) activation which is involved in the transforming growth of B lymphocytes and has been studied as being a driving force for EBV gene expression in tumors $[8,9,11]$.

LMP1 also functions in protecting the cell from apoptosis by inducing the expression of the B-cell lymphoma 2 (BCL-2) gene as well as inhibiting the metastasis suppressors, such as RE version inducing cysteine rich protein with kazal motifs 1 (RECK1) and Ecadherin, and promoting angiogenesis through the induction of angiogenic factors such as the hypoxiainducible factor 1- $\alpha$ (HIF1?), vascular endothelial growth factor (VEGF),fibroblast growth factor 2(FGF2), and the Cyclooxygenase-2 gene (COX2) [8,10,12-14]. Furthermore, it regulates the expression of Matrix Metalloproteases (MMP), such as MMP9 or MMP1 which are essential for the reorganization and degradation of the extracellular matrix that precedes the invasion [15]. Recently it has also been discovered that the LMP1 acts as a molecule modulating cell adhesion via the regulation of activin-A/TGF-? and integrin ?1 signalling [16].

LMP1 has been described as an active receptor and is known as an equivalent to the CD40 receptor belonging to the TNF family since both have homologous functions [17]. Based on this property, LMP1 was able to partially replace CD40 in transgenic mice lacking CD40 [18,19]. Also, it cannot be disregarded, when studying pathologies associated with EBV, the fact that the signalling complexes engaged by the LMP1 may depend on the cells type. A new property concerning the oncogenicity of LMP1 has been demonstrated by the He team in 2016, and it is the upregulation of the expression of the protein

\section{Virology \& Immunology Journal}

$\mathrm{TAZ}$ which is an essential protein for the proliferation of cells [20].

During latency, a truncated form of LMP1 is found. The expression of LMP1, in general, is correlated with a poor survival prognosis for patients with NPC [21]. Several studies have tried to target this protein because of its major oncogenic power and, most recently, a promising preclinical study in a mouse model has shown that a therapeutic vaccine targeting the LMP1 protein could suppress tumor growth and metastasis in vivo [22].

LMP-1 regulates and promotes glycolysis through many pathways, genes, gene regulators, tumor suppressors, and enzymes such as PI3-K/Akt, Ras, mTOR, HIF-1 $\alpha$, c-Myc, NF-кB, p53, GSK3beta, FBW7, FGFR1, the M2 splice isoform of pyruvate kinase PKM2, PDHK1, Hexokinase $2 \mathrm{HK} 2$, VHL, and mutations of isocitrate dehydrogenase 1 (IDH1), succinate dehydrogenase (SDH), and fumarate hydratase (FH) [2-4].

In order to achieve such excessive division during tumorigenesis in NPC, EBV-infected cells need energy. Recent studies have discussed how LMP1 mediate energy metabolism reprograming in these cells, by activating multiple signaling pathways leading to more aerobic glycolysis.

In this Review, the authors discuss the most recent literature on how LMP1 alter the signaling pathways of Glycolysis during NPC pathogenesis.

\section{LMP1/NF-кB/ Glycolysis Signaling Pathways}

NF-kappaB (NF- $\mathrm{kB}$ ) pathway is formed of a family of five eukaryotic nuclear transcription factors: RelA (p65), RelB and c-Rel, and the precursor proteins NF- $\mathrm{kB} 1$ (p105) and NF- $\mathrm{KB} 2$ (p100), which are processed into p50 and p52, respectively [23]. This pathway has an important role in controlling many cellular processes (cell growth, proliferation, survival, death and apoptosis, response to cellular stresses, physiological aspects of immune and inflammatory responses, regulating genes expression...). In addition, it is involved in several diseases (cancer, arthritis, chronic inflammation, asthma, neurodegenerative diseases, and heart disease) [23].

In normal cells, NF-kB complexes (NF- $\kappa \mathrm{B}$ bound to I $\mathrm{B}$ proteins) are present in the cytoplasm in a latent inactive form, when activated the complexes enter the nucleus and activate gene expression. In mutated cells, the complex is located in the nucleus in an active form $[24,25]$. 
$\mathrm{NF}-\mathrm{\kappa B}$ is an important signaling pathway activated by the EBV-encoded LMP1, in normal cell NF- $\mathrm{BB}$ can be activated through two distinct pathways: the canonical pathway (major or classical pathway) and the noncanonical pathway (minor or alternative pathway) $[26,27]$.

LMP1 cytoplasmic carboxyl tail: C-terminal activating regions (CTAR1 and CTAR2) [28]. CTAR1 and CTAR2 contribute with different abilities in NF- $\mathrm{BB}$, mTORC1, AKT, MEK, ERK, and IKK (IKK $\alpha$ and IKK $\beta$ ) activation, in addition to Glut-1 transcription [26,29-33].

In NPC, CTAR1 of LMP1 has a leading role in activating AKT, MEK, and ERK and also induce IKK $\alpha$ phosphorylation. On the other hand, CTAR2 of LMP1 has a leading role in activating mTORC1 through IKK $\beta$ mediated phosphorylation of Ser939 of tuberous sclerosis TSC2, as well as inducing Glut-1 transcription. However, mTORC1 signaling pathway could also be activated by phosphatidylinositol 3-kinase (PI3K)/AKT [29,34,35].

Hence, both expression of LMP1 activates mTORC1 signaling and NF- $\kappa \mathrm{B}$ transcription in nucleus cells, the canonical pathway where CTAR2 is the key modulator, through the phosphorylation of IKK $\alpha$ by IKB kinase $\beta$ (IKK $\beta$ ), and the nuclear translocation of the p65/p50 dimer complex. The non-canonical pathway where CTAR1 is the key modulator, activates NF- $\mathrm{KB}$ transcription in the nucleus, through the phosphorylation of $\operatorname{IKK} \beta$, which mediates processing of $\mathrm{p} 100$-RelB by NIK, and generates the nuclear translocation of the p52/RelB dimer [36].

Thus, and in case of NPC mTORC1/ NF- $\mathrm{kBsignaling} \mathrm{by}$ LMP1 plays an essential role in modulating glucose metabolism [37]. mTORC1 is known for supporting cell growth by regulating energy metabolism. Furthermore LMP1, mTORC1, NF-kB, and Glut-1 interact to regulate aerobic glycolysis. As previouslymentioned, LMP1 activates mTORC1 which induces NF- $\mathrm{KB}$ signaling activation and Glut-1 transcription [38]. mTORC1/ NF- $\kappa B$ signaling in LMP1-expressing cells accelerates glucose uptake, lactate production and aerobic glycolysis [29]. The principal of the previously stated pathways is illustrated in figure 1.

\section{LMP1/C-MYC/ Glycolysis Signaling Pathways}

C-Myc is a transcriptional factor and an antiproliferative genes inhibitor. This multifaceted oncogene also known as "master regulator" regulates many aspects of both processes: Cellular growth and Cellular metabolism.

\section{Virology \& Immunology Journal}

Its control can be both direct (increase of energetic supplies) and indirect (regulation of lipid, glutamine and glucose metabolism, mitochondrial and ribosomal biosynthesis) [39].

c-Myc has an important function in tumors such as Burkitt's lymphoma. In addition to being a downstream "early response" gene, it responds to the activation of many signaling pathways [40].

Studies has shown that C-Myc regulates many glucose metabolisms via genes direct regulation, such as: GLUT1, Hexokinase 2 (HK2), phosphofructokinase (PFKM), and Enolase1 (ENO1) [41,42]. By regulating these genes, it has been known that c-Myc participates directly to the Warburg Effect [1]. During Hypoxia, ENO1 stimulates MBP1 to initiate a translation, then MBP1 regulates negatively C-Myc expression $[43,44]$.

Studies on mice have confirmed C-Myc's direct effect on glycolytic activity. In fact, overproduction of lactic acid and high glycolytic enzyme activity were found in mice's liver facing overexpressed c-Myc. On the other hand, overexpression ofLDH-A alone was found in transfected rodent fibroblasts, while those transformed by C-Myc overproduce lactate. This suggests that Warburg Effect can be induced by LDH-A (a downstream target of C-Myc) $[45,46]$.

GLUT-1 proteins facilitate the import of glucose across the lymphocyte cell membrane. The levels of glucose can be regulated by some tumor suppressors and oncogenes, such as C-Myc and RAS that induce GLUT-1 mRNA, but p53 suppresses the expression of GLUT1,3 and 4, while PI3K increases the expression of GLUT-1 and GLUT-3 [47]. C-Myc was affirmed to be an essential transcriptional factor for LMP1 induced upregulation of HK2 and enhancement of glycolysis in NPC cells, and the LMP1-irritated PI3K/Akt-GSK3beta-FBW7 signaling axis results in settlement of C-Myc in NPC cells [48]. All of the previously stated is illustrated in figure I.

Despite the fact that the fundamental instruments of the deregulation of glucose digestion in malignancy cells have not yet been totally illustrated, some tumor related genes such as c-Myc and HIF-1-alfa are known to take an interest in regulating energy metabolic reprogramming in malignancy cells. Decreased c-Myc levels conduct to a PTEN overexpression and gives cancer resistance by negatively controlling PI3-K/Akt pathway [49,50].

An unknown mechanism has been revealed recently for the LMP-1 upregulation of C-Myc and it also recognize 


\section{Virology \& Immunology Journal}

a PI2K/Akt-GSK3beta-FBW7- C-Myc signaling axis proving that when LMP-1 is present in NPC cells it increases glycolysis. Due to EBV infection, metabolic changes in cells will occur. In fact, the capacity of LMP-1 to induce glycolytic catalysis adds to the noteworthiness of EBV in glucose digestion in NPC cells [48].

The PI3K/Akt signal pathway acts for the most part by disturbing the stabilization of C-Myc. At the point when PI3K/Akt-GSK3beta-FBW7/c-Myc signaling axis is obstructed, C-Myctransactive the transcription of HK2, which causes an upregulation of glycolysis and presents a negative regulation on mitochondrial-dependent apoptosis, which relates the general survival of NPC patients [48]. The principal of the previously stated pathways is illustrated in figure I.

\section{LMP1/Glut- 1/ Glycolysis Signaling Pathways}

Glut-1 is a member of the glucose transporters family (Glut-1 to -4), which transports extracellular glucose into the cell across the plasma membranes, it is very low expressed in normal cells [1]. This transporter is overexpressed in many tumors, including hepatic, pancreatic, breast, esophageal, and many other types of cancer [51]. Glut-1 regulates aerobic glycolysis, cell growth, proliferation, apoptosis, colony formation, and anchorage-independent growth of LMP1-expressing cells [29]. Now it's already established that an over-expression of GLUT mRNA and GLUT transporter are associated with diverse type on human cancer cell $[52,53]$.

LMP1 promotes aerobic glycolysis by increasing glucose uptake. Furthermore, it regulates Glut-1 transcription (mRNA and protein of Glut-1) through mTORC1 activation and PI3K/AKT, NF- $\kappa B$, IKK $\beta$, AKT signaling. Glut-1 gene is a direct target gene of NF- $\kappa B$ signaling [29].

Jing Chen et al found that the mTOR signaling pathway is regulated by LMP1 expression in NPC. LMP1 and the genes in the mTOR pathway such as p-P70S6K and p4EBP1 may be potential prognostic biomarkers [37].

Some transcription factors like Sp1 and HIF- $1 \alpha$ also regulate Glut-1 transcription. The C-terminal activating region 2 (CTAR2) of LMP1, plays an essential role in mTORC1 activation, mainly through IKK -mediated phosphorylation of TSC2 at Ser939. Moreover,mTORC1 regulates energy metabolism, and LMP1-induced NF- $\mathrm{\kappa B}$ activation and Glut-1 transcriptionin NPC cells [29,54]. The principal of the previously stated pathways is illustrated in figure I.

\section{LMP1/ Hypoxia-Inducible Factor (HIF)/ Glycolysis Signaling Pathways}

Hypoxia-inducible factor (HIF), a central regulator for detecting and adapting to cellular oxygen levels, transcriptionally activates genes modulating oxygen homeostasis and metabolic activation [55]. Beyond this, HIF influences many other processes. Hypoxia, in part through HIF-dependent mechanisms, influences epigenetic factors, including DNA methylation and histone acetylation, which modulate hypoxia-responsive gene expression in cells [55].

It has shown that the principal EBV oncoprotein, latent membrane protein 1 (LMP1), activates HIF1A and subsequently expression of HIF1-responsive genes in epithelial cells [56].

LMP1 up-regulates the level of Siah1 E3 ubiquitin ligase by enhancing its stability, which subsequently induces proteasomal degradation of prolyl HIFhydroxylases 1 and 3 that normally mark HIF1A for degradation [56].

Wakisaka et al report that expression of HIF- $1 \alpha$ is increased in diverse Epstein-Barr virus (EBV)-infected cell lines, which express EBV latent membrane protein 1 (LMP1), but not in the parental EBV-negative cell lines, suggesting that LMP1 increases HIF-1 activity through induction of HIF-1 $\alpha$ protein expression, which is controlled by $\mathrm{p} 42 / \mathrm{p} 44$ MAPK activity and $\mathrm{H}_{2} \mathrm{O}_{2}$. The ability of EBV, and specifically its major oncoprotein, LMP1, to induce HIF-1 $\alpha$ along with other invasiveness and angiogenic factors reported previously discloses additional oncogenic properties of this protein in NPC cells [57].

Wei-Wen Sung et al show that under LMP1 excitation, remarkable activity of ERK1/2 signaling in HIF- $1 \alpha$ gene transcription through preponderant action of NF- $\mathrm{KB}$ p50 was shown. In addition, they prove that LMP1 activate transcription and post-transcription processes of the HIF$1 \alpha$ gene in NPC cells [58]. In the post-transcription step, the stability of HIF-1 $\alpha$ mRNA was enhanced via downregulation of RNA-destabilizing TTP and PUM2 by LMP1 through CTAR1- and CTAR3-engaged ERK1/2, as well as the STAT3 signaling pathway [58]. Concomitantly, the LMP1 CTAR1-recruited ERK1/2 signal was also involved in the facilitation of the HIF-1A promoter by LMP1 through activation of the canonical NF- $\mathrm{kB}$ pathway. Notably, elevated HIF- $\alpha$ further displayed a positive feedback loop of transcription regulation on its own gene promoter under LMP1 excitation [58]. The principal of the previously stated pathways is illustrated in figure I. 


\section{Conclusion}

Most oncogenic viruses, including EBV has their oncoprotein, these oncoprotein help viruses to establishes a latent growth-transforming infection in host cells. This review described and illustrated the main signaling pathways that LMP1 can alter in order to activate aerobic glycolysis. The authors shown that LMP1 activates mTORC1 which induces NF- $\mathrm{KB}$ signaling activation and Glut-1 transcription [38]. mTORC1/ NF- $\mathrm{KB}$ signaling in LMP1-expressing cells accelerates glucose uptake, lactate production and aerobic glycolysis [29]. In parallel LMP1 induce and activate C-Myc witch is an essential for the upregulation of HK2 and enhancement of glycolysis, and the LMP1-irritated PI3K/Akt-GSK3beta-FBW7 signaling axis results in settlement of C-Myc in NPC cells [48]. Furthermore, LMP1 enhance the activity of ERK1/2 signaling in HIF-1 $\alpha$ gene transcription through preponderant action of NF- $\mathrm{kB}$ p50. In addition, this review describes how LMP1 activate transcription and post-transcription processes of the HIF- $1 \alpha$ gene in NPC cells [58]. In the post-transcription step, the stability of HIF-1 $\alpha$ mRNA was enhanced via down-regulation of RNA-

\section{Virology \& Immunology Journal}

destabilizing TTP and PUM2 by LMP1 through CTAR1and CTAR3-engaged ERK1/2, as well as the STAT3 signaling pathway [58].

In conclusion, LMP1 is a key regulator of the reprogramming of EBV-mediated glycolysis especially in NPC cells. Given the importance of EBV-mediated deregulation of glycolysis, anti-glycolytic therapy might represent a worthwhile avenue of exploration in the treatment of EBV-related cancers. Understanding the overall mechanism of aerobic glycolysis, then the ability of the oncoprotein of a tumor virus to alter and modify these signaling pathways, can help to develop an antiglycolytic therapy which could be an encouraging opportunity in the management of EBV-related cancer [48].

Lastly, adding the capacity of EBV to induce aerobic glycolysis to its relevant role to induce cell proliferation and the inhibition of apoptosis, all these characteristics should prompt current research to improve the vaccination strategies against EBV.

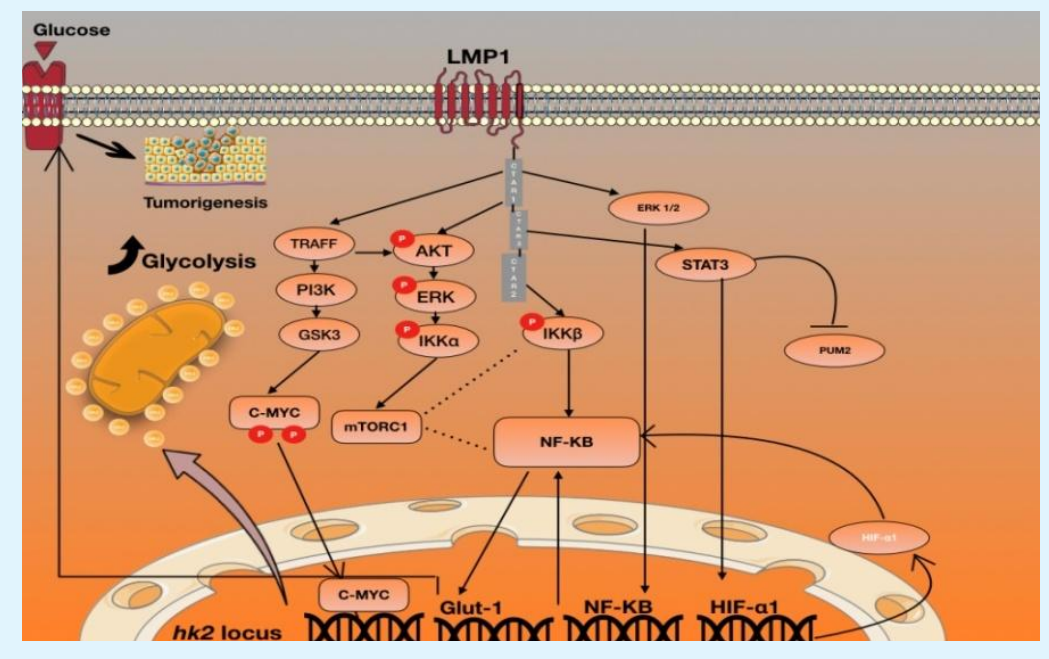

Figure: Schematic Illustration of LMP1-induced glycolysis pathways inEBV-related carcinoma cells.

\section{References}

1. Potter M, Newport E, Morten KJ (2016) The Warburg effect: 80 years on. Biochem Soc Trans 44(5): 14991505.

2. Sung WW, Chen PR, Liao MH, Lee JW (2017) Enhanced aerobic glycolysis of nasopharyngeal carcinoma cells by Epstein-Barr virus latent membrane protein 1. Exp Cell Res 359(1): 94-100.

3. Miller DM, Thomas SD, Islam A, Muench D, Sedoris K (2012) c-Myc and cancer metabolism. Clin Cancer Res 18(20): 5546-5553.

4. Lo AK, Dawson CW, Young LS, Ko CW, Hau PM, et al. (2015) Activation of the FGFR1 signalling pathway by the Epstein-Barr virus-encoded LMP1 promotes 


\section{Virology \& Immunology Journal}

aerobic glycolysis and transformation of human nasopharyngeal epithelial cells. J Pathol 237(2): 238248.

5. Peh SC, Sandvej K, Pallesen G (1995) Epstein-Barr virus (EBV) in Malaysian upper-aerodigestive-tract lymphoma: incidence and sub-type. Int J Cancer 61(3): 327-332.

6. Chang $\mathrm{MH}, \mathrm{Ng} \mathrm{CK}$, Lin YJ, Liang CL, Chung PJ, et al. (1997) Identification of a promoter for the latent membrane protein 1 gene of Epstein-Barr virus that is specifically activated in human epithelial cells. DNA Cell Biol 16(7): 829-837.

7. Fennewald S, van Santen V, Kieff E (1984) Nucleotide sequence of an mRNA transcribed in latent growthtransforming virus infection indicates that it may encode a membrane protein. J Virol 51(2): 411-419.

8. Dawson CW, Port RJ, Young LS (2012) The role of the EBV-encoded latent membrane proteins LMP1 and LMP2 in the pathogenesis of nasopharyngeal carcinoma (NPC). Semin Cancer Biol 22(2): 144-153.

9. Senyuta N, Goncharova E, Kondratova V, Scherback L, Smirnova K, et al. (2015) Epstein-Barr virus status and LMP1 oncogene polymorphism in patients with nasopharyngeal carcinoma and other tumors of the oral cavity in a non-endemic region. In: Kondratova LS, Smirnova K, Vayradyan V, Lomaya M, Maksimovich D (Eds.) Neck cancer. Epidemiology, Management and Treatment Outcomes. Nova Science Publishers, New York, pp: 101-121.

10. Laherty CD, Hu HM, Opipari AW, Wang F, Dixit VM (1992) The Epstein-Barr virus LMP1 gene product induces A20 zinc finger protein expression by activating nuclear factor kappa B. J Biol Chem 267(34): 24157-24160.

11. Chen H, Lee JM, Zong Y, Borowitz M, Ng MH, et al. (2001) Linkage between STAT regulation and Epstein-Barr virus gene expression in tumors. Journal of virology 75(6): 2929-2937.

12. Banko A, Lazarevic I, Cupic M, Stevanovic G, Boricic I, et al. (2012) Carboxy-terminal sequence variation of LMP1 gene in Epstein-Barr-virus-associated mononucleosis and tumors from Serbian patients. J Med Virol 84(4): 632-642.

13. da Costa VG, Marques-Silva AC, Moreli ML (2015) The Epstein-Barr virus latent membrane protein-1 (LMP1) 30-bp deletion and Xhol-polymorphism in nasopharyngeal carcinoma: a meta-analysis of observational studies. Syst Rev 4: 46.

14. A H, George T, Vm K, S MA (2008) Arteriovenous malformation after transradial percutaneous coronary intervention. Indian Heart J 60(1): 64.

15. Murono S, Yoshizaki T, Sato H, Takeshita H, Furukawa $M$, et al. (2000) Aspirin inhibits tumor cell invasiveness induced by Epstein-Barr virus latent membrane protein 1 through suppression of matrix metalloproteinase- 9 expression. Cancer Res 60(9): 2555-2561.

16. Morris MA, Dawson CW, Laverick L, Davis AM, Dudman JPR, et al. (2016) The Epstein-Barr virus encoded LMP1 oncoprotein modulates cell adhesion via regulation of activin A/TGFbeta and beta1 integrin signalling. Sci Rep 6: 19533.

17. Eliopoulos AG, Rickinson AB (1998) Epstein-Barr virus: LMP1 masquerades as an active receptor. Curr Biol 8(6): R196-198.

18. Uchida J, Yasui T, Takaoka-Shichijo Y, Muraoka M, Kulwichit W, et al. (1999) Mimicry of CD40 signals by Epstein-Barr virus LMP1 in B lymphocyte responses. Science 286(5438): 300-303.

19. Hömig-Hölzel C, Hojer C, Rastelli J, Casola S, Strobl LJ, et al. (2008) Constitutive CD40 signaling in B cells selectively activates the noncanonical NF-kappaB pathway and promotes lymphomagenesis. J Exp Med 205(6): 1317-1329.

20. He J, Tang F, Liu L, Chen L, Li J, et al. (2016) Positive regulation of TAZ expression by EBV-LMP1 contributes to cell proliferation and epithelialmesenchymal transition in nasopharyngeal carcinoma. Oncotarget 8(32): 52333-52344.

21. Mao Y, Lu MP, Lin H, Zhang DW, Liu Y, et al. (2013) Prognostic significance of EBV latent membrane protein 1 expression in lymphomas: evidence from 15 studies. PLoS One 8(4): e60313.

22. Lin M-C, Lin Y-C, Chen S-T, Young T-H, Lou P-J (2017) Therapeutic vaccine targeting Epstein-Barr virus latent protein, LMP1, suppresses LMP1-expressing tumor growth and metastasis in vivo. BMC Cancer 17(1): 18.

23. Oeckinghaus A, Hayden MS, Ghosh S (2011) Crosstalk in NF-kappaB signaling pathways. Nat Immunol 12(8): 695-708. 
24. Zhang Q, Lenardo MJ, Baltimore D (2017) 30 Years of NF-kappaB: A Blossoming of Relevance to Human Pathobiology. Cell 168(1-2): 37-57.

25. Schulze-Luehrmann J, Ghosh S (2006) Antigenreceptor signaling to nuclear factor kappa $\mathrm{B}$. Immunity 25(5): 701-715.

26. Gewurz BE, Mar JC, Padi M, Zhao B, Shinners NP, et al. (2011) Canonical NF-kappaB activation is essential for Epstein-Barr virus latent membrane protein 1 TES2/CTAR2 gene regulation. J Virol 85(13): 67646773.

27. Liu T, Zhang L, Joo D, Sun SC (2017) NF-kappaB signaling in inflammation. Signal Transduct Target Ther 2 .

28. Eliopoulos AG, Young LS (2001) LMP1 structure and signal transduction. Semin Cancer Biol 11(6): 435444.

29. Zhang, J, Jia L, Lin W, Yip YL, Lo KW, et al. (2017) Epstein-Barr Virus-Encoded Latent Membrane Protein 1 Upregulates Glucose Transporter 1 Transcription via the mTORC1/NF-kappaB Signaling Pathways. J Virol 91(6).

30. Chen J (2012) Roles of the PI3K/Akt pathway in Epstein-Barr virus-induced cancers and therapeutic implications. World J Virol 1(6): 154-161.

31. Dawson CW, Tramountanis G, Eliopoulos AG, Young LS (2003) Epstein-Barr virus latent membrane protein 1 (LMP1) activates the phosphatidylinositol 3-kinase/Akt pathway to promote cell survival and induce actin filament remodeling. J Biol Chem 278(6): 3694-3704.

32. Kung CP, Meckes DG Jr, Raab Traub N (2011) EpsteinBarr virus LMP1 activates EGFR, STAT3, and ERK through effects on PKCdelta. J Virol 85(9): 4399-4408.

33. Lavorgna A, Harhaj EW (2012) EBV LMP1: New and shared pathways to NF-kappaB activation. Proc Natl Acad Sci U S A 109(7): 2188-2189.

34. Huen DS, Henderson SA, Croom-Carter D, Rowe M (1995) The Epstein-Barr virus latent membrane protein-1 (LMP1) mediates activation of NF-kappa B and cell surface phenotype via two effector regions in its carboxy-terminal cytoplasmic domain. Oncogene 10(3): 549-560.

\section{Virology \& Immunology Journal}

35. Shair KH, Bendt KM, Edwards RH, Bedford EC, Nielsen $\mathrm{JN}$, et al. (2007) EBV latent membrane protein 1 activates Akt, NFkappaB, and Stat3 in B cell lymphomas. PLoS Pathog 3(11): e166.

36. Hayden MS, Ghosh S (2008) Shared principles in NFkappaB signaling. Cell 132(3): 344-362.

37. Chen J, Hu CF, Hou JH, Shao Q, Yan LX, et al. (2010) Epstein-Barr virus encoded latent membrane protein 1 regulates mTOR signaling pathway genes which predict poor prognosis of nasopharyngeal carcinoma. J Transl Med 8: 30.

38. Sommermann TG, O'Neill K, Plas DR, Cahir-McFarland E (2011) IKKbeta and NF-kappaB transcription govern lymphoma cell survival through AKT-induced plasma membrane trafficking of GLUT1. Cancer Res 71(23): 7291-7300.

39. Levens D (2010) You Don't Muck with MYC. Genes Cancer 1(6): 547-554.

40. Teicher BA, Linehan WM, Helman LJ (2012) Targeting cancer metabolism. Clin Cancer Res 18(20): 55375545 .

41. Kim JW, Gao P, Liu YC, Semenza GL, Dang CV (2007) Hypoxia-inducible factor 1 and dysregulated c-Myc cooperatively induce vascular endothelial growth factor and metabolic switches hexokinase 2 and pyruvate dehydrogenase kinase 1 . Mol Cell Biol 27(21): 7381-7393.

42. Kim JW, Tchernyshyov I, Semenza GL, Dang CV (2006) HIF-1-mediated expression of pyruvate dehydrogenase kinase: a metabolic switch required for cellular adaptation to hypoxia. Cell Metab 3(3): 177-185.

43. Sedoris KC, Thomas SD, Miller DM (2010)Hypoxia induces differential translation of enolase/MBP-1. BMC Cancer 10: 157.

44. Hanahan D, Weinberg RA (2011) Hallmarks of cancer: the next generation. Cell 144(5): 646-674.

45. Shim H, Dolde C, Lewis BC, Wu CS, Dang G, et al. (1997) c-Myc transactivation of LDH-A: implications for tumor metabolism and growth. Proc Natl Acad Sci U S A 94(13): 6658-6663.

46. Valera A, Pujol A, Gregori X, Riu E, Visa J, et al. (1995) Evidence from transgenic mice that myc regulates hepatic glycolysis. FASEB J 9(11): 1067-1078. 


\section{Virology \& Immunology Journal}

47. Piccaluga PP, Weber A, Ambrosio MR, Ahmed Y, Leoncini L (2018) Epstein-Barr Virus-Induced Metabolic Rearrangements in Human B-Cell Lymphomas. Front Microbiol 9: 1233.

48. Xiao L, Hu Z-y, Dong X, Tan Z, Li W, et al. (2014) Targeting Epstein-Barr virus oncoprotein LMP1mediated glycolysis sensitizes nasopharyngeal carcinoma to radiation therapy. Oncogene 33(37): 4568-4578.

49. Chen JQ, Russo J (2012) Dysregulation of glucose transport, glycolysis, TCA cycle and glutaminolysis by oncogenes and tumor suppressors in cancer cells. Biochim Biophys Acta 1826(2): 370-384.

50. Garcia-Cao I, Song MS, Hobbs RM, Laurent G, Giorgi C, et al. (2012) Systemic elevation of PTEN induces a tumor-suppressive metabolic state. Cell 149(1): 4962.

51. Medina RA, Owen GI (2002) Glucose transporters: expression, regulation and cancer. Biol Res 35(1): 926.

52. Yamamoto T, Seino Y, Fukumoto H, Koh G, Yano H, et al. (1990) Over-expression of facilitative glucose transporter genes in human cancer. Biochem Biophys Res Commun 170(1): 223-230.
53. Brown RS, Wahl RL (1993) Overexpression of Glut-1 glucose transporter in human breast cancer. An immunohistochemical study. Cancer 72(10): 29792985.

54. Szablewski L (2013) Expression of glucose transporters in cancers. Biochim Biophys Acta 1835(2): 164-169.

55. Choudhry H, Harris AL (2018) Advances in HypoxiaInducible Factor Biology. Cell Metab 27(2): 281-298.

56. Kondo S, Seo SY, Yoshizaki T, Wakisaka N, Furukawa $\mathrm{M}$, et al. (2006) EBV latent membrane protein 1 upregulates hypoxia-inducible factor 1alpha through Siah1-mediated down-regulation of prolyl hydroxylases 1 and 3 in nasopharyngeal epithelial cells. Cancer Res 66(20): 9870-9877.

57. Wakisaka N, Kondo S, Yoshizaki T, Murono S, Furukawa M, et al. (2004) Epstein-Barr virus latent membrane protein 1 induces synthesis of hypoxiainducible factor 1 alpha. Mol Cell Biol 24(12): 52235234.

58. Sung WW, Chu YC, Chen PR, Liao MH, Lee JW (2016) Positive regulation of $\mathrm{HIF}-1 \mathrm{~A}$ expression by EBV oncoprotein LMP1 in nasopharyngeal carcinoma cells. Cancer Lett 382(1): 21-31. 